\title{
FOOD INDUCED ESTERASE PHENOCOPIES IN THE SNAIL CEPAEA NEMORALIS
}

\author{
G. S. OXFORD \\ Department of Biology, University of York, Heslington, York YOI SDD
}

Received 23.v.75

\begin{abstract}
SUMMARY
Hepatopancreatic extracts from the snail Cepaea nemoralis, assayed straight from the field, often contain three or four heavily staining esterase zones which migrate to the cathodal end of polyacrylamide disc gels during electrophoresis. Previous breeding results showed that the heavily straining zones appeared allelic but to incorporate these multibanded phenotypes, a super gene of five closely linked loci was tentatively proposed. Further breeding work again failed to demonstrate multiple zones in parents or offspring and so experiments were conducted to see whether the multi-zoned phenotypes in the wild were produced by secondary modification of single primary products. Wild snails yielding extracts containing more than two heavily staining zones were shown to possess only two such zones after three months under laboratory conditions. Also, the ingestion of nettle (Urtica dioica L.) has been demonstrated to induce extra esterase zones in laboratory-reared animals. Some of the secondarily induced zones appear identical in physical, biochemical and electrophoretic properties to the primary products of other alleles, and thus appear to be electrophoretic phenocopies. A model is suggested which could account for this phenomenon.
\end{abstract}

\section{Introduction}

In the past two decades there has been an explosive increase in the use of zone electrophoresis as a technique for measuring genetic variation in a wide variety of organisms (Harris, 1966; Lewontin and Hubby, 1966; Selander and Yang 1969; Marshall and Allard, 1970). It is usually assumed that enzyme bands revealed on electrophoretic gels represent the primary product of a locus and that the variations in pattern observed between individuals result from the presence of alleles coding for electrophoretically distinct allozymes.

In the majority of cases interpretations along these lines are probably correct but occasionally they can lead to a totally false model of the genetic basis underlying isozyme production. The present paper is a preliminary report of just such a system; a series of esterase zones derived from the hepatopancreas of the land snail Cepaea nemoralis (L.).

\section{The enzyme system}

General methods of tissue extraction, polyacrylamide disc electrophoresis and the subsequent staining of esterase zones have been described elsewhere (Oxford, 1973b). The esterases under consideration here give rise to the most cathodally migrating zones called Est. 1, Est. 2, Est. 3, Est. 4 and 
Est. 5, the latter zone migrating the farthest from the origin (fig. 1). All of the esterases exhibit the same physical and biochemical properties (Oxford, $1971,1973 a, c)$ and under laboratory conditions may be strongly staining, weakly staining or absent. In extracts taken from individuals freshly caught in the wild this distinction between strongly and weakly staining zones may be slightly blurred although on most gels differential staining intensities are clear. Phenotypes and genotypes are denoted in a shorthand form. Thus 1-3 means that Est. 1 and Est. 3 are strongly staining.

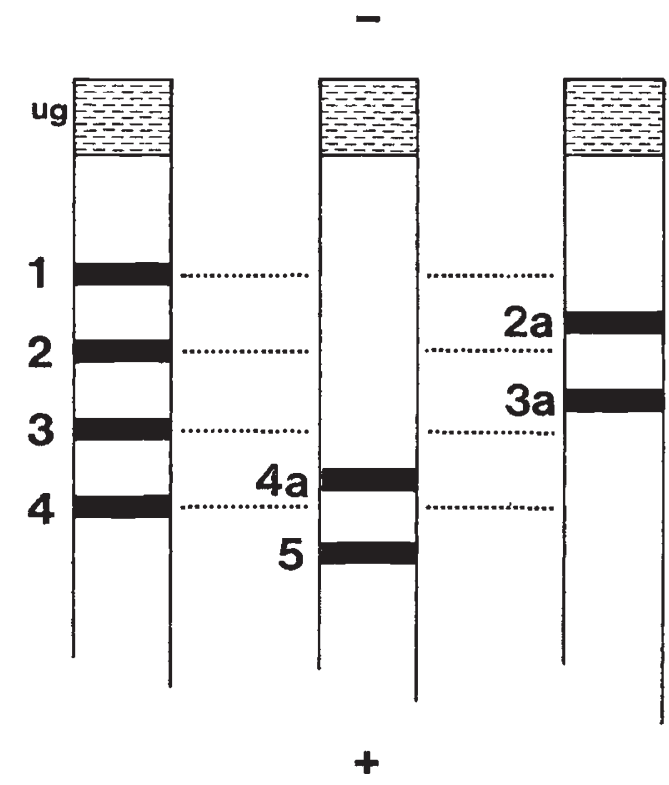

FIG. 1.-Diagram of the most cathodal esterase zones as they appear on 4 per cent polyacrylamide gels. $u g=$ upper gel. Migration is from top to bottom.

Results from the earlier breeding experiments (Oxford, 1973b) showed that strongly staining esterase zones Est. 1, Est. 2, Est. 3 and Est. 4 are coded for by alleles and that at each position in the gel occupied by an enzyme the strongly staining zones are dominant to weakly staining or absent zones. Subsequent breeding has demonstrated that another two zones, Est. 2a migrating to a position between Est. 1 and Est. 2 and Est. 4 a migrating to a position between Est. 3 and Est. 4 (fig. 1) are also probably determined by alleles at this locus (table 1). A recent re-examination of the esterases of snails retained from the first breeding programme and of photographs taken at the time indicate that in at least two cases (matings $\mathrm{M}$ and $\mathrm{P}$ (Oxford, 1973b)) the zone scored as Est. 4 was in fact Est. 4a. Samples were then being run on 7.5 per cent acrylamide gels in which the difference in migration between Est. 4 and Est. $4 \mathrm{a}$ is very small. All subsequent analyses have been performed on 4 per cent acrylamide gels which give a much larger separation of these zones. The possibility exists that all of the zones described as Est. 4 in the early work were Est. 4 a, which leaves the genetic status of Est. 4 in some doubt.

Analysis of material from the field showed that individuals could possess 
more than two heavily staining zones, an impossible situation if the single locus model is correct. Also, extracts from laboratory bred snails often produce lightly staining zones at positions not occupied by heavily staining zones. This again is not consistent with the single locus hypothesis.

After considering various two and three locus models the tentative hypothesis finally suggested (Oxford, 1973b) was that five loci are involved, $E s-1$ to $E s-5$ (Est. 2a and Est. 4 a were not then recognized; Est. 5 was included in the system because it has identical physical and biochemical properties to Est. 1 to 4 and because it appears to be in linkage disequilibrium

TABLE 1

Inheritance of Est. $2 a$ and Est. $4 a$.

\begin{tabular}{|c|c|c|c|c|c|c|c|c|c|c|c|c|c|}
\hline \multirow[b]{2}{*}{ Brood } & \multicolumn{2}{|c|}{ Parents } & \multicolumn{7}{|c|}{ FI genotypes } & \multirow[b]{2}{*}{$\mathbf{N}$} & \multirow[b]{2}{*}{$x^{2}$} & \multirow[b]{2}{*}{ d.f. } & \\
\hline & 1 & 2 & 1 & 3 & $1-3$ & $2 a-3$ & $1-4 a$ & $3-4 a$ & $2 a-4 a$ & & & & \\
\hline N2 & 1 & $\times 1-4 a$ & 12 & - & - & - & 12 & - & - & 24 & 0 & 1 & n.s. \\
\hline N22 & & $\times 3-4 a$ & - & - & 17 & - & 14 & - & - & 31 & 0.145 & 1 & n.s. \\
\hline $\mathrm{X} 4$ & $2 a-3$ & $\times 3-4 a$ & - & 7 & - & 5 & - & 9 & 9 & 30 & 1.467 & 3 & n.s. \\
\hline
\end{tabular}

Source of parents: N2/I Barbury castle b, Marlborough (for map ref. see table 5).

N2/2 Ridgeway 1, Marlborough (SU141762).

N22/1 and N22/2 Ridgeway 1 .

$\mathrm{X} 4 / 1$ and X4/2 Newborough Warren, Anglesey (SH431639).

with these zones in the field). Each locus was thought to be responsible for esterase activity at one level in the gel. As no cross-over products had been detected in the breeding programme it could be assumed that the loci were closely linked and that at each locus three alleles were present to account for the three levels of staining activity found in laboratory bred animals.

The presence of the esterase supergene had only been inferred and so further breeding experiments were started using snails from colonies on the Marlborough Downs, Wiltshire known to contain high proportions of animals exhibiting more than two heavily staining zones. When these parents were analysed after egg laying, all were shown to possess only one or two heavily staining zones in this region of the gel, exactly like the individuals used in the previous breeding programme (Oxford, 1973b). Snails which had been kept in the laboratory for many months as parents and those which had been bred under these conditions never showed the three or four heavily staining zones frequently detected in individuals assayed straight from the wild. This suggested that some aspect of the laboratory environment may have been influencing the expression of this esterase system.

\section{The EXPERIMENTS}

\section{(i) Preliminary experiment}

The first experiment involved the analysis of batches of snails after different periods of laboratory culture. A sample of 52 Cepaea nemoralis was collected in June 1974 from a hedgerow near Grimston Hill, York. This snail sample was divided into three subsamples, the first of which $(N=25)$ was extracted and electrophoresed one day after collection from the wild. 
The rest of the sample was kept in two large plastic boxes floored with paper tissue and maintained at $19^{\circ} \mathrm{C}$. Snails were fed on carrot slices and calcium was provided in the form of natural chalk. A second subsample $(\mathrm{N}=13)$ was removed randomly from both boxes and assayed after 19 days. The remainder $(N=14)$ were processed after a total of 97 days in the laboratory.

The object of this experiment was to see whether esterase patterns which were inconsistent with a single locus model i.e. those with more than two heavily staining zones, persisted under laboratory conditions. Snail zymograms were therefore scored as having more than two heavily staining zones or as having two zones or less. The results are shown in table 2. Samples analysed after one and 19 days in the laboratory were identical within sampling error ( $\mathrm{P}=0 \cdot 8$, Fisher's exact test-one tail since deviations in only one direction are expected) and so the scores can be amalgamated. In the

TABLE 2

The effect of laboratory conditions on the esterases of three snail batches

\begin{tabular}{cccc} 
Days after & \multicolumn{2}{c}{ Number of snails with } & \\
\cline { 2 - 3 } 1 & $\leqslant 2$ heavy zones & $>2$ heavy zones & Total \\
19 & 19 & 6 & 25 \\
97 & 9 & 4 & 13 \\
14 & 0 & 14
\end{tabular}

subsample assayed after 97 days no individual had more than two heavily staining zones, a situation which is significantly different from the previous subsamples $(P=0.03)$. There is evidence here that the laboratory environment can affect the esterase phenotype to the extent that the number of heavily staining zones is reduced to one or two.

\section{(ii) Changes in individual snails in the laboratory}

The second experiment was similar to the first except that the phenotypic changes induced by laboratory conditions were followed in individual snails so that the pattern of alteration could be identified. In the summer of 1974 a total of 447 snails were collected from 11 colonies on the Marlborough Downs, Wiltshire. Animals were kept in an aestivating condition in dry cardboard boxes until extracts could be made. Of the snails caught 318 were assayed for their esterase phenotypes by levering off the very tip of the shell spire and removing the most apical portion of hepatopancreas thus exposed. This was then extracted in the usual way (Oxford, 1973b). All snails were processed within 13 days of collection although the extracts were analysed some time later. It has been shown that in extract form at $-20^{\circ} \mathrm{C}$ esterase patterns are completely stable for at least 12 months (Oxford, 1971). Operated snails were individually marked on their shells and maintained under the same conditions as those in experiment one except that lettuce was also supplied. In most cases new shell was laid down within 2 or 3 days of the operation, sealing the exposed hepatopancreas.

After 6 to 7 months the surviving snails were re-extracted and rescored for their Est. 1 to 5 phenotypes. Of the 313 snails operated on (five were too small to extract in this way and were killed so that the whole of their hepatopancreas could be used) 170 were still alive at the end of the experiment, an 


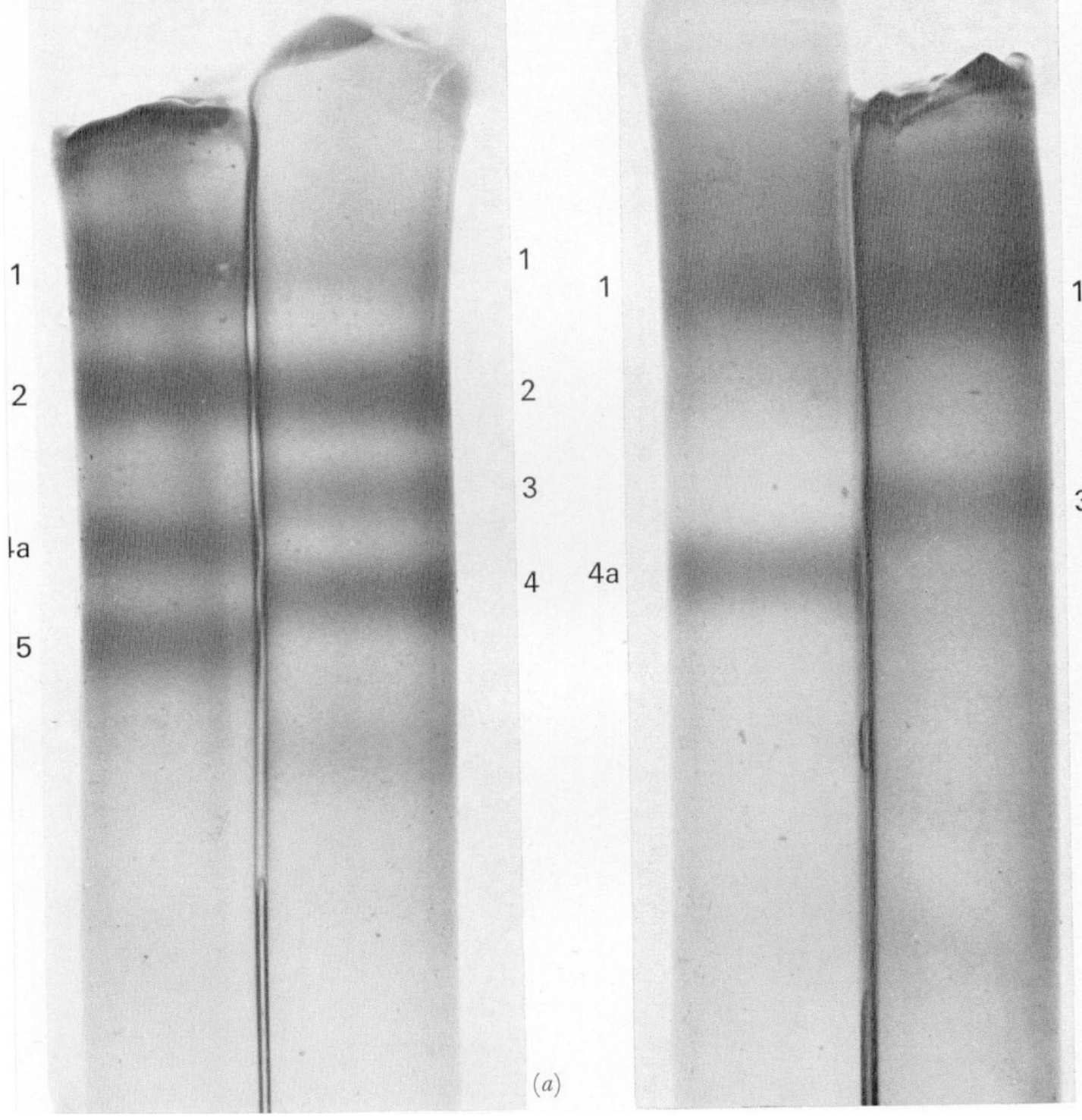

Plate I

Esterase zones derived from two snails assayed straight from the wild (a) and again after 6 months in the laboratory $(b)$. Note the reduction in the number of heavily staining esterase zones from four to two. 
overall mortality of $45 \cdot 8$ per cent. Most of the individuals that died did so within the first few days after the operation because new shell failed to develop and the hepatopancreas frequently became infected with fungus. There is no evidence that mortality was in any way connected with particular esterase phenotypes. For technical reasons eleven snails were not rescored.

Comparisons of esterase phenotypes scored soon after collection and again after 6 months in the laboratory confirmed the results of the previous experiment. Initially, extracts from many snails showed three or four heavily staining esterase zones on electrophoresis whereas later extracts from the same individuals contained only two such zones. The detailed results from this experiment are given in table 3 and some of the phenotype changes which occurred are shown in plate I.

TABLE 3

Esterase phenotypes of snails assayed straight from the wild and phenotypes after 6 months in the laboratory

\begin{tabular}{|c|c|c|}
\hline $\begin{array}{c}\text { Wild } \\
\text { phenotype }\end{array}$ & $\begin{array}{l}\text { Laboratory } \\
\text { phenotype }\end{array}$ & No. tested \\
\hline $1-2$ & 1 & 25 \\
\hline 2 & 1 & 40 \\
\hline $1-2$ & $1-2$ & 6 \\
\hline 2 & $1-2$ & 21 \\
\hline $1-2-3$ & $1-2$ & 5 \\
\hline $2-3$ & $1-2$ & 6 \\
\hline 2 & 2 & 1 \\
\hline $2-3$ & 2 & 4 \\
\hline $2-3$ & $2-3$ & 1 \\
\hline $2-3-4$ & $2-3$ & 1 \\
\hline $3-4$ & $2-3$ & 1 \\
\hline $2-3-4$ & $1-3$ & 6 \\
\hline $1-2-3$ & $1-3$ & 1 \\
\hline $1-2-3-4$ & $1-3$ & 13 \\
\hline $2-4$ & $1-3$ & 5 \\
\hline $3-4$ & 3 & 4 \\
\hline 4 & 3 & 1 \\
\hline $1-2 a-2-3 a$ & $1-2 a$ & 1 \\
\hline $1-2-4 a-5$ & $1-4 a$ & 6 \\
\hline $2-4 a-5$ & $1-4 a$ & 4 \\
\hline $2-5$ & $1-4 a$ & 2 \\
\hline $2-4 a-5$ & $2-4 a$ & 1 \\
\hline $3-4 a-4-5$ & $3-4 a$ & 2 \\
\hline $4-5$ & $3-4 a$ & 1 \\
\hline & & al 158 \\
\hline
\end{tabular}

Experiments one and two were concerned with the reduction in the number of heavily staining esterase zones observed in animals brought into the laboratory. The third experiment attempted to reverse the process i.e. to induce new zones in snails reared in the laboratory by exposing them to different environmental conditions.

(iii) Esterase changes under different nutritional conditions

The esterases under consideration are only found in the hepatopancreas whereas all other esterases in this organ are also found in the kidney (Oxford, 
1971). This distribution suggests a tissue specific function for these enzymes and although the hepatopancreas is involved with many functions other than digestion e.g. excretion and calcium metabolism (Abolins-Krogis, 1961), the first variables tested were different foods and substrates.

Immature progeny of parents N22 (table 1) were assigned to four experimental cages, 12 snails per cage. The only food experienced by these animals since hatching was carrot, lettuce and occasionally a mixture of powdered oats, chalk and dried milk. The cages consisted of large insect rearing cylinders and were furnished as follows:

\begin{tabular}{|c|c|c|}
\hline Cage & Substrate & Food \\
\hline 2 & Tissue paper & Carrots and lettuce \\
\hline 4 & Soil & Carrots and lettuce \\
\hline 1 & Soil & $\begin{array}{l}\text { Nettle plants (Urtica } \\
\text { dioica L.) }\end{array}$ \\
\hline 3 & Soil & $\begin{array}{l}\text { Nettle, groundsel (Senecio } \\
\text { vulgaris L.), plantain } \\
\text { Plantago major L.) and } \\
\text { coarse grass }\end{array}$ \\
\hline
\end{tabular}

Cage two mimicked typical laboratory culture conditions while cage three aimed at simulating conditions experienced by snails in the wild although these will vary greatly from colony to colony. The other cages, one and four, were intermediate between these two. All cages were maintained at $19^{\circ} \mathrm{C}$ and exposed to $18: 6$ hour light: dark cycle. Food plants grew in the

TABLE 4

The numbers of each esterase phenotype found in the four experimental cages

$\begin{array}{lrrrr}\begin{array}{l}\text { Esterase } \\ \text { phenotype }\end{array} & 2 & 4 & 3 & 1 \\ 1-3 & 9 & 4 & 3 & 2 \\ 1-4 a & 1 & 8 & - & 1 \\ 2-5 & - & - & 2 & 3 \\ 1-2-4 a-5 & - & - & 1 & 1 \\ \begin{aligned} \text { C-2-4a-5 } \\ 2-4\end{aligned} & - & - & - & 1 \\ 2-3-4 & - & - & - & 2 \\ \begin{array}{l}\text { P-4a } \\ \text { Totals }\end{array} & - & - & - & 1 \\ \begin{array}{c}\text { Mean hepatopancreas } \\ \text { score }\end{array} & 10 & - & -- & 1 \\ & 0.60 & 0.92 & 2.50 & 3.75\end{array}$

soil whereas carrot and lettuce were changed weekly. Cages were sprayed with water twice weekly and each contained calcium in the form of natural chalk lumps.

After 86 days the experiment was terminated because of an unexplained high mortality in cage three. Snails were extracted in the normal way and at the same time the colour of the hepatopancreas was determined as this has been shown to reflect the type of food ingested by Cepaea (Oxford, 1971). Tissue pellets left in the centrifuge tubes after extraction were compared with a preserved set of standard hepatopancreas samples prepared from wild and 
laboratory reared snails. Samples of these glands were spun down in centrifuge tubes and the pellets preserved in 5 per cent formalin. Colours of the standards ranged from black (score 4) through three intermediates to a pale buff (score 0) (Oxford, 1971).

Mean hepatopancreas scores and the esterase phenotypes in each of the four cages are shown in table 4 . The ingestion of soil and nettle both lead to higher hepatopancreas scores (Oxford, 1971) which suggests that snails from cage four had eaten some soil and that individuals in cage three had eaten plants other than nettle. Esterase phenotypes not present in snails reared on carrot and lettuce were generated in animals occupying the cages containing nettle as a food plant; three from six in cage three and eight from twelve in cage one had altered esterase patterns. This experiment not only shows that the changes in esterase phenotype are completely reversible but that they can be induced by the ingestion of nettle. Ingestion of soil substrate appears to have no effect on this esterase system.

\section{Discussion}

The preliminary experiment indicated that under laboratory conditions esterase phenotypes consisting of more than two heavily staining zones do not persist. As no individual died during the course of the experiment this cannot have been due to the selective deaths of snails with multibanded esterase phenotypes and must have resulted from secondary alterations within particular animals. This finding was confirmed by the second experiment. In extracts of snails which have been kept in the laboratory for 3 months or more only one or two heavily staining esterase zones are found (but see below). The results from the breeding programmes indicate that the zones which persist in the laboratory are genetically determined and are probably coded for by alleles (table 1 and Oxford, 1973b). Since the " extra" zones detected in wild caught snails have been shown to be phenotypically generated, the main reason for postulating that the esterases in this system are controlled by a supergene of five loci (Oxford, 1973b) is removed. It now seems more likely that only one locus is involved.

The precise relationship between the esterase phenotypes exhibited by snails assayed straight from the wild and the genotypes shown when the same animals have been kept under laboratory conditions for 6 months is complex (table 3). Four generalisations can be made, however. First, the secondarily induced zones are always more anodal than the primary zones from which they appear to be derived. Thus, if an individual is homozygous for the allele coding for Est. 2, Est. 3 is generated in the wild but Est. 1 is not. Second, only the next most anodal zone is produced from each primary zone in the wild. For example, if Est. 2 is generated from Est. 1 it does not, in turn, lead to the production of a heavily staining Est. 3, at least not under the conditions studied so far. Third, both primary and induced zones may persist in the wild giving a four-banded pattern in heterozygotes. On the other hand, one or both primary zones may be fully converted into induced zones giving three- and two-banded phenotypes respectively. Lastly, because the secondary product of one allele can coincide in position with the primary product of another, apparently identical phenotypes can be produced by different genotypes and, vice versa, the same genotypes can 
give rise to different phenotypes. In many cases therefore, it is impossible to score the genotype of an individual from the phenotype it yields in the wild.

In four cases genetic information can be gleaned from a knowledge of the phenotype pattern. The secondary zones are always more anodal than the primary zones from which they appear to be derived. It follows therefore that any phenotype which exhibits a strongly staining Est. 1 will contain the allele coding for this zone-it cannot, according to the present scheme, be produced in any other way. The second zone which can give information on the genotype if present in the phenotype is Est. 5. This esterase seems to be produced only as a secondary product of Est. $4 \mathrm{a}$. The presence, therefore, of Est. $4 \mathrm{a}$ and/or Est. 5 in an esterase phenotype indicates the presence of the allele coding for Est. 4a. Similarly the presence of zones Est. 2a and/or Est. $3 a$ (the esterase generated from it) indicates that the individual is carrying

\section{Table 5}

Allele frequencies at the Es-1 locus in nine colonies of Cepaea nemoralis on the Marlborough Downs, Wiltshire

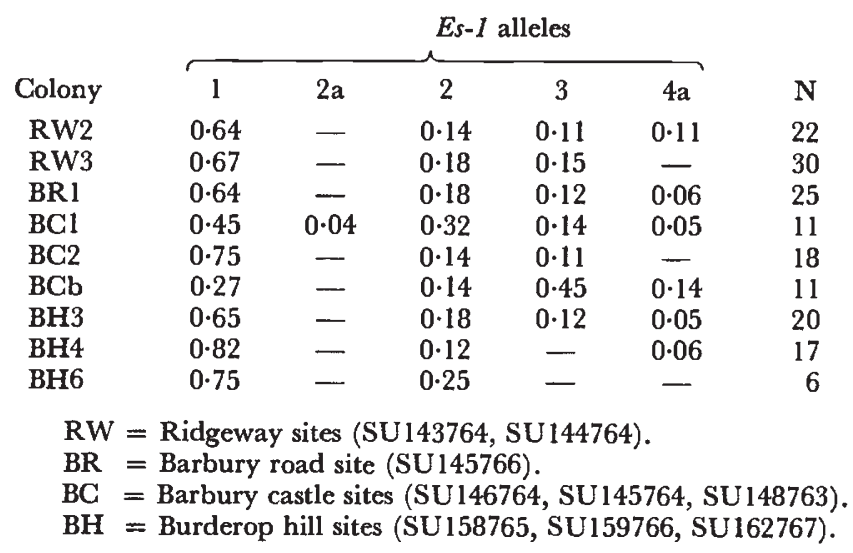

the allele for Est. 2a. Finally, as Est. 4, as a primary product, appears to be absent on the Marlborough Downs, animals exhibiting this zone must contain the allele for Est. 3.

It was argued above that the esterases under consideration here are probably coded for by alleles. The locus is $E s-1$ and the alleles $E s-1^{1}$, $E_{s-1} 1^{2 a}, E s-1^{2}, E s-1^{3}, E s-1^{4 a}$ and possibly $E s-1^{4}$ producing Est. 1, Est. 2a, Est. 2, Est. 3, Est. 4a and Est. 4 respectively. This nomenclature supersedes that used by Oxford (1973b). One snail in experiment two exhibited a "genotype" which is not possible according to the single locus hypothesis. After 6 months in the laboratory the phenotype of this individual had remained unchanged viz. 1-2-3. There is no explanation for this occurrence at present. Table 5 gives allele frequencies in colonies on the Marlborough Downs from which snails were removed for use in experiment two.

The third experiment cited above gives information on the nature of the environmental conditions which induce secondary esterases in Cepaea nemoralis. It showed that the presence of nettle (Urtica dioica) as a food plant could lead to the production of extra esterase zones. When nettle was the only food available the degree of conversion was greater than when other 
vegetation was present. An examination of hepatopancreas scores under different food régimes suggests that the group of snails with the highest proportion of secondary esterase zones had ingested the highest amount of nettle. It is not known what exactly it is in nettle that is responsible for this effect or whether nettle is the only plant capable of inducing extra esterase zones. All of the colonies investigated on the Marlborough Downs were found in habitats containing at least some nettle plants but there is no obvious correlation between the proportion of individuals showing secondary enzyme zones and the amount of nettle available to them. Further experiments are needed to assess how much nettle needs to be ingested before secondary esterase zones are induced and to determine how long these esterases persist if no more nettle is eaten.

The relative distances between Est. 1 and Est. 2, Est. 2 and Est. 3 and Est. 3 and Est. 4 on gels are the same suggesting a constant charge difference between the molecules. Considering the allelic nature of these zones, it is likely that this constant charge difference is a unit difference, produced by the substitution of a single neutral (or positively charged) amino acid for a negatively charged (or neutral) one. Est. $4 \mathrm{a}$ and Est. $2 \mathrm{a}$ are shifted slightly towards the cathode with respect to Est. 4 and Est. 2. The cathodal shift is the same in both cases and the secondary zones generated from them (Est. 5 and Est. 3a, respectively) have exactly the same spatial relationship to their "parental" zones as is shown by Est. 1, Est. 2, Est. 3 and Est. 4 (fig. 1).

One may conjecture that, while the primary products of different alleles differ in single amino acid replacements involving charged residues, the secondary modifications (all, apparently resulting in a unit increase in negative charge) may be due to deamidation of a vulnerable glutamine or aparagine residue or to the blocking of an amino group by acetylation (or formylation) (see, for example, Terhorst et al., 1973).

Although the heavily staining esterase zones in Cepaea nemoralis can be explained on the basis of one-step modification, the occasional presence of weaker staining esterase zones requires a more elaborate hypothesis. For example, if a snail is homozygous for allele $E s-1^{1}$ then both Est. 1 and the derived Est. 2 may stain heavily on electrophoretic gels. Often, however, traces of Est. 3 and occasionally Est. 4 can be discerned. These are possibly produced in very small amounts from Est. 2 by additional modifications. Further speculation is not worthwhile until more information becomes available.

Just how widespread enzyme phenocopies of this kind are is hard to determine. In a high proportion of studies in which gel electrophoresis is used to score genetic variation in organisms no attempt is made to determine the genetic control of the enzyme zones so revealed. In some cases, of course, this is impossible. Even when genetic analysis is attempted erroneous conclusions may be reached if the existence of phenocopies is not suspected, as in the earlier work on the genetics of this esterase system in Cepaea (Oxford, $1973 b)$. Even when the secondary zones are not phenocopies of primary zones one might be led seriously astray by interpreting two bands as two alleles without taking into account the possibility that they might be environmentally modified products of a single allele.

Acknowledgments.-I am grateful to Drs S. J. S. Hardy and J. C. Sparrow for their comments on various drafts of the manuscript. I should also like to thank my wife for her assistance in collecting snails from the Marlborough Downs. 


\section{G. S. OXFORD}

\section{REFERENGES}

ABOLINŠ-KROGIS, A. 1961. The histochemistry of the hepatopancreas of Helix pomatia in relation to the regeneration of the shell. Ark. Zool., 13, 159-201.

harris, H. 1966. Enzyme polymorphism in man. Proc. Roy. Soc. Lond. B, 164, 298-310.

LEWONTIN, R. C., AND HUBBY, J. L. 1966. A molecular approach to the study of genetic heterozygosity in natural populations. II. Amount of variation and degree of heterozygosity in natural populations of Drosophila pseudoobsura. Genetics, 54, 595-609.

MARSHALL, D. R., AND ALLARD, R. W. 1970. Isozyme polymorphisms in natural populations of Avena fatua and Avena barbarta. Heredity, 29, 373-382.

OXFORD, G. s. 1971. The properties, genetics and ecogenetics of esterases in Cepaea. Unpublished Ph.D. thesis, University of Liverpool.

OXFORD, G. S. 1973a. The molecular weight relationships of esterases in Cepaea nemoralis and C. hortensis (Mollusca:Helicidae) and their genetical implications. Biochem. Genet., 8, 365-382.

Oxpord, G. s. 1973b. The genetics of Cepaea esterases. 1. Cepaea nemoralis. Heredity, 30, 127-139.

OXFORD, G. s. 1973c. The biochemical properties of esterases in Cepaea (Mollusca: Helicidae). Comp. Biochem. Physiol., 45B, 529-538.

SELANDER, R. K., AND YANG, S. Y. 1969. Protein polymorphism and genic heterozygosity in a wild population of the house mouse (Mus musculus). Genetics, 63, 653-667.

TERHORST, G. P., MÖLLER, W., LAURSEN, R., AND WITTMANN-LIEBOLD, B. 1973. The primary structure of an acidic protein from 50-S ribosomes of Escherichia coli which is involved in GTP hydrolysis dependent on elongation factors G and T. Eur. F. Biochem., 34, 138-152. 